\title{
Longitudinal coherence in thermal ghost imaging
}

\author{
F. Ferri, ${ }^{\text {a) }}$ D. Magatti, V. G. Sala, and A. Gatti \\ CNR-INFM-CNISM Dipartimento di Fisica e Matematica, Università dell'Insubria, Via Valleggio 11, \\ Como I-22100, Italy
}

(Received 24 April 2008; accepted 27 May 2008; published online 1 July 2008)

\begin{abstract}
We show theoretically and experimentally that lensless ghost imaging with thermal light is fully interpretable in terms of classical statistical optics. The disappearance of the ghost image when the object and the reference planes are located at different distances from the source is due to the fading out of the intensity-intensity cross correlation between the two planes. Thus the visibility and the resolution of the ghost image are determined by the longitudinal coherence of the speckle beam, and no quantum explanation is necessary. (C) 2008 American Institute of Physics.
\end{abstract}

[DOI: 10.1063/1.2945642]

Ghost imaging and ghost diffraction with thermal light have attracted over the past decade a lot of attention in the field of quantum optics. The main reason is probably related to the fact that, although they were originally discovered ${ }^{1,2}$ by exploiting the quantum properties of entangled photon pairs, they can also be observed with a thermal source and explained in terms of classical optics. The equivalence between the quantum and the classical descriptions was demonstrated theoretically ${ }^{3}$ and verified in a number of different experiments by using nonthermal ${ }^{4}$ and thermal sources. ${ }^{5-7}$ Nevertheless, part of the scientific community seems to be still puzzled by this duality and, whenever some unexpected results are not immediately understandable in terms of classical optics, often the quantum nature of light is invoked. ${ }^{8-10}$ And, this dichotomy brings new fuel to the discussion. ${ }^{11}$

An example of this debate are two letters recently appeared in this journal. ${ }^{12,13}$ In work, ${ }^{12}$ the group of Scarcelli et al. reports on a lensless ghost imaging experiment carried by using a pseudothermal source realized by shining a laser beam onto a rotating ground glass. The authors observed a clear ghost image of the object (a double slit) in the in-focus condition, i.e., when the distance $z_{1}$ between the object and the source is equal to the distance $z_{2}$ between the source and the reference plane, where the image is reconstructed. When the out-of-focus condition $\left(z_{1} \neq z_{2}\right)$ was investigated, the ghost image was highly blurred and the double slit was indistinguishable. They attributed this effect to the quantum nature of two-photon interference, which, quoting their work, was stated as ".... the observation of ghost imaging with thermal light is a quantum two-photon interference effect." It should be noted that the authors did not provide enough optical details of the source and did not characterized the speckle fields at the object and image planes. As a consequence, it is not clear what was the level of correlation between the intensities at the two planes.

In the second work, ${ }^{13}$ Basano and Ottonello repeated the same experiment under similar conditions, but in this case the thermal source clearly produced far-field speckles, which remain correlated over very long distances. ${ }^{4,15}$ Thus, they observed a neat ghost image also in the out-of-focus condition, and the overall results were (correctly) interpreted in terms of classical optics. However, these authors did not give

${ }^{a)}$ Author to whom correspondence should be addressed. Electronic mail: fabio.ferri@uninsubria.it. any explanation for the reason why the authors of Ref. 12 do not see the image when $z_{1} \neq z_{2}$.

In this letter we reproduce the same experiment, and show that, by controlling the longitudinal spatial coherence properties of the thermal source, it is possible to provide a fully classical explanation of the results obtained by the two groups. In particular, we show, theoretically and experimentally, that, also in the out-of-focus condition, the resolution of the ghost image is set by the width $2 \sigma$ of the intensityintensity cross-correlation function of the speckle beams at the planes $z_{1}$ and $z_{2}$. As explained in Ref. 15, this width increases with the distance $\Delta z=z_{2}-z_{1}$ at a rate which can be fairly rapid in the case of small speckles generated close to the source. Thus, when $2 \sigma$ becomes comparable with the length scales of the object, the ghost image gets blurred: this is what presumably happens in the experiment of Ref. 12 . Conversely, far away from the source, the far-field speckles are much broader and remain correlated over much longer distances. ${ }^{14,15}$ Thus the ghost image can be accurately retrieved also for large distances $\Delta z$, as it clearly happens in the case of Ref. 13.

The experimental setup is sketched in Fig. 1. The pseudothermal source ${ }^{16}$ emits at $\lambda=0.532 \mu \mathrm{m}$ and produces a collimated beam of speckles whose transversal size, at the $z=0$ plane, is $\delta_{0} \sim 45 \mu \mathrm{m}$. On this plane the beam is transversely limited by a diaphragm of diameter $D=5.5 \mathrm{~mm}$ and the source behaves as a planar quasihomogeneous source (see Ref. 16 for details). Speckles generated in the proximity of this source are the so-called near-field speckles, ${ }^{15,17,18}$ whose size does not change with the distance $z$. For this source the crossover between the near-field and far-field regimes ${ }^{17}$ occurs at a distance $z_{\mathrm{NF}} \sim D \delta_{0} / \lambda \sim 465 \mathrm{~mm}$. Thus for $z \ll z_{\mathrm{NF}}$, the transversal speckle size is constant $[\delta(z)$ $\left.\sim \delta_{0}\right]$ and the longitudinal distance $\Delta z$ over which the speckles remain correlated is $\sim \pi \delta_{0}^{2} / \lambda \sim 10 \mathrm{~mm}$. $^{15,16}$ The speckle

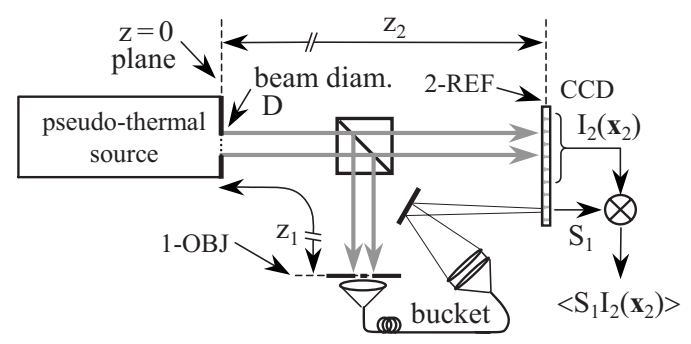

FIG. 1. Schematic diagram of the setup. 
beam is divided by a cube beam splitter into two "twin" beams, the transmitted (ref arm) and the reflected (obj arm) beam. The object is placed on the obj arm at a fixed distance $z_{1}=200 \mathrm{~mm}$ from the source. The intensity distribution $I_{2}\left(\mathbf{x}_{2}\right)$ in the ref arm is measured at a distance $z_{2}$ by using a twodimensional (2D) charge coupled device (CCD) sensor, whose (squared) pixel size is $6.67 \mu \mathrm{m} \ll \delta_{0}$. The object is a double slit made of a rectangular aperture, $690 \mu \mathrm{m}$ wide-900 $\mu \mathrm{m}$ high, inside which a thin needle of $160 \mu \mathrm{m}$ diameter is inserted. The object area is therefore $A_{\text {obj }}$ $\sim 0.48 \mathrm{~mm}^{2}$. The bucket detection is carried out by collecting all the light passing trough the object with lens coupled with a multimode optical fiber whose output is refocused onto a small area of the CCD [nonoverlapped to $I_{2}\left(\mathbf{x}_{2}\right)$ ] and integrated so to retrieve the bucket signal $S_{1}$

Let us indicate with $I_{1}\left(\mathbf{x}_{1}\right)$ and $I_{2}\left(\mathbf{x}_{2}\right)$, the intensity distributions of the speckle fields on the obj and ref planes located, respectively, at distances $z_{1}$ and $z_{2}$ from the thermal source. If $t(\mathbf{x})$ indicates the (field) object transmission function, the bucket signal $S_{1}$ is

$$
S_{1}=\int I_{1}\left(\mathbf{x}_{1}\right)\left|t\left(\mathbf{x}_{1}\right)\right|^{2} d \mathbf{x}_{1} .
$$

If we assume uniform illumination so that $\left\langle I_{1}\left(\mathbf{x}_{1}\right)\right\rangle=\left\langle I_{1}\right\rangle$ $(\langle\cdot\rangle=$ ensemble average over different realizations of the speckle field), the average value of $S_{1}$ is

$$
\left\langle S_{1}\right\rangle=\left\langle I_{1}\right\rangle \int\left|t\left(\mathbf{x}_{1}\right)\right|^{2} d \mathbf{x}_{1}=\left\langle I_{1}\right\rangle A_{\text {obj }}
$$

in which $A_{\text {obj }}$ is equal to the object area when $|t(\mathbf{x})|^{2}$ is a step function equal to 1 inside the object and 0 outside. The correlation between $S_{1}$ and $I_{2}\left(\mathbf{x}_{2}\right)$ is

$$
\left\langle S_{1} I_{2}\left(\mathbf{x}_{2}\right)\right\rangle=\int\left\langle I_{1}\left(\mathbf{x}_{1}\right) I_{2}\left(\mathbf{x}_{2}\right)\right\rangle\left|t\left(\mathbf{x}_{1}\right)\right|^{2} d \mathbf{x}_{1},
$$

which depends on the intensity-intensity correlation function $\left\langle I_{1}\left(\mathbf{x}_{1}\right) I_{2}\left(\mathbf{x}_{2}\right)\right\rangle$. The latter one can be expressed in terms of the Siegert relation ${ }^{14}$

$$
\left\langle I_{1}\left(\mathbf{x}_{1}\right) I_{2}\left(\mathbf{x}_{2}\right)\right\rangle=\left\langle I_{1}\left(\mathbf{x}_{1}\right)\right\rangle\left\langle I_{2}\left(\mathbf{x}_{2}\right)\right\rangle\left[1+\left|\mu_{z_{1}, z_{2}}\left(\mathbf{x}_{1}, \mathbf{x}_{2}\right)\right|^{2}\right]
$$

in which

$$
\mu_{z_{1}, z_{2}}\left(\mathbf{x}_{1}, \mathbf{x}_{2}\right)=\frac{\left\langle A_{1}^{*}\left(\mathbf{x}_{1}\right) A_{2}\left(\mathbf{x}_{2}\right)\right\rangle}{\sqrt{\left\langle I_{1}\left(\mathbf{x}_{1}\right)\right\rangle\left\langle I_{2}\left(\mathbf{x}_{2}\right)\right\rangle}}
$$

is the cross-correlation coefficient of the electric fields $A_{1}\left(\mathbf{x}_{1}\right)$ and $A_{2}\left(\mathbf{x}_{2}\right)$ at the planes $z_{1}$ and $z_{2}$, respectively. Since for our setup $z_{1}, z_{2} \ll z_{\mathrm{NF}}$, the function $\mu$ depends only on the differences $\Delta \mathbf{x}=\mathbf{x}_{2}-\mathbf{x}_{1}$ and $\Delta z=z_{2}-z_{1}{ }^{15,17}$ Thus, recalling the hypothesis of uniform illumination, Eq. (4) becomes

$$
\left\langle I_{1}\left(\mathbf{x}_{1}\right) I_{2}\left(\mathbf{x}_{2}\right)\right\rangle=\left\langle I_{1}\right\rangle\left\langle I_{2}\right\rangle\left[1+\left|\mu_{\Delta z}(\Delta \mathbf{x})\right|^{2}\right] .
$$

The function $\mu_{\Delta z}(\Delta \mathbf{x})$ is normalized to unity $\left(\left|\mu_{0}(0)\right|^{2}=1\right)$ and, for $\Delta z=0$, coincides with the complex coherence factor $\mu_{0}(\Delta \mathbf{x}) \cdot{ }^{14}$ The latter one defines the coherence area

$$
A_{\mathrm{coh}}=\int\left|\mu_{0}(\Delta \mathbf{x})\right|^{2} d \Delta \mathbf{x},
$$

which is related to the speckle size by $\delta \sim \sqrt{A_{\text {coh }}}$. Another property of $\mu_{\Delta z}$ is ${ }^{15}$ fixed $z_{1}=200 \mathrm{~mm}$ and varying $z_{2}=z_{1}+\Delta z$. The result $\mu_{\Delta z}$ is
Author complimentary copy. Redistribution subject to AIP license or copyright, see http://apl.aip.org/apl/copyright.jsp

$$
\int\left|\mu_{\Delta z}(\Delta \mathbf{x})\right|^{2} d \Delta \mathbf{x}=\int\left|\mu_{0}(\Delta \mathbf{x})\right|^{2} d \Delta \mathbf{x},
$$

which is therefore a conserved quantity, independent of $\Delta z$. If we indicate with $2 \sigma_{\mu}(\Delta z)$ the rms full width of $\left|\mu_{\Delta z}\right|^{2}$, being the integral of $\left|\mu_{\Delta z}\right|^{2}$ constant, its width increases as

$$
2 \sigma_{\mu}(\Delta z) \sim 2 \sigma_{\mu}(0) /\left|\mu_{\Delta z}(0)\right| .
$$

Thus $2 \sigma_{\mu}(\Delta z)$ is minimum for $\Delta z=0$ and increases as the inverse of the axial amplitude $\left|\mu_{\Delta z}(0)\right|$.

Returning to ghost correlation, by using Eq. (6), we can write Eq. (3) as

$$
\left\langle S_{1} I_{2}\left(\mathbf{x}_{2}\right)\right\rangle=\left\langle S_{1}\right\rangle\left\langle I_{2}\right\rangle+\left\langle I_{1}\right\rangle\left\langle I_{2}\right\rangle \int\left|\mu_{\Delta z}\left(\mathbf{x}_{2}-\mathbf{x}_{1}\right)\right|^{2}\left|t\left(\mathbf{x}_{1}\right)\right|^{2} d \mathbf{x}_{1}
$$

showing that $\left\langle S_{1} I_{2}(x)\right\rangle$ depends on $\Delta z$ through the convolution between $\left|\mu_{\Delta z}\right|^{2}$ and $|t|^{2}$. At this point is convenient to introduce the function

$$
\gamma_{\Delta z}(\mathbf{x})=\frac{\left|\mu_{\Delta z}(\mathbf{x})\right|^{2}}{\int\left|\mu_{\Delta z}(\mathbf{x})\right|^{2} d \mathbf{x}}=\frac{\left|\mu_{\Delta z}(\mathbf{x})\right|^{2}}{A_{\text {coh }}}
$$

so that $\int \gamma_{\Delta z}(\mathbf{x}) d \mathbf{x}=1$. By combining Eq. (10) and the right hand side of Eq. (11), we arrive to the final result, which written in terms of relative fluctuations, is

$$
\nu_{\Delta z}\left(\mathbf{x}_{2}\right) \equiv \frac{\left\langle\delta S_{1} \delta I_{2}\left(\mathbf{x}_{2}\right)\right\rangle}{\left\langle S_{1}\right\rangle\left\langle I_{2}\right\rangle}=\frac{\left|t\left(\mathbf{x}_{2}\right)\right|^{2} \otimes \gamma_{\Delta z}\left(\mathbf{x}_{2}\right)}{N_{\mathrm{obj}}},
$$

where $\otimes$ represents the (transversal) 2D-convolution product, and $N_{\mathrm{obj}}=A_{\mathrm{obj}} / A_{\mathrm{coh}}$ is the number of coherence areas falling inside the object. Equation (12) shows the two key features of thermal ghost imaging carried out with a bucket detection: (a) the spatial resolution of the ghost image is set by the convolving function $\gamma_{\Delta z}(\Delta \mathbf{x})$, whose (transversal) width (the same as that of $\left|\mu_{\Delta z}\right|^{2}$ ) is given by Eq. (9); thus the best resolution is achieved for $\Delta z=0$ and becomes progressively deteriorated as $\Delta z$ is increased; (b) the visibility [i.e., the maximum value of $\left.\nu_{\Delta z}\left(\mathbf{x}_{2}\right)\right]$ is equal to $1 / N_{\text {obj }}$ and, except for convolution effects (occurring when $\gamma_{\Delta z}(\Delta \mathbf{x})$ and $t(\Delta \mathbf{x})$ have similar widths), is independent of $\Delta z$.

The first experiment was carried out in the in-focus condition, namely, $z_{1}=z_{2}=200 \mathrm{~mm}$. The results are reported on the first raw of Fig. 2 where panel (a1) shows the 2D-ghost image retrieved by averaging $M=10^{4}$ independent realizations of the speckle field. Panel (b1) shows the horizontal profile of the double slit, obtained by averaging all the lines of panel (a1) falling within the object (continuous line); this profile compares quite well with the expected one (dotted line) and its visibility $\left(\sim 3.4 \times 10^{-3}\right)$ is fairly consistent with Eq. (12). Panel (c1) represents the 2D-square modulus of $\left|\mu_{\Delta z}\right|^{2}$, obtained by removing the object from the obj arm and cross correlating $I_{1}\left(\mathbf{x}_{1}\right)$ and $I_{2}\left(\mathbf{x}_{2}\right)$. The radial profile of $\left|\mu_{\Delta z}\right|^{2}$, reported in panel (d1), has an amplitude $\sim 1$ (as expected for highly contrasted speckles) and a rms width $2 \sigma_{\mu}$ $\sim 45 \mu \mathrm{m}$, much smaller than the linear object size. Correspondingly, the double slit is retrieved with high spatial resolution. Note that, since $z_{1}=z_{2}$, the full width of $\left|\mu_{0}(\Delta x)\right|^{2}$ corresponds to the speckle size, i.e., $\delta \sim 2 \sigma_{\mu}$.

The out-of-focus condition was investigated by keeping fixed $z_{1}=200 \mathrm{~mm}$ and varying $z_{2}=z_{1}+\Delta z$. The results for 

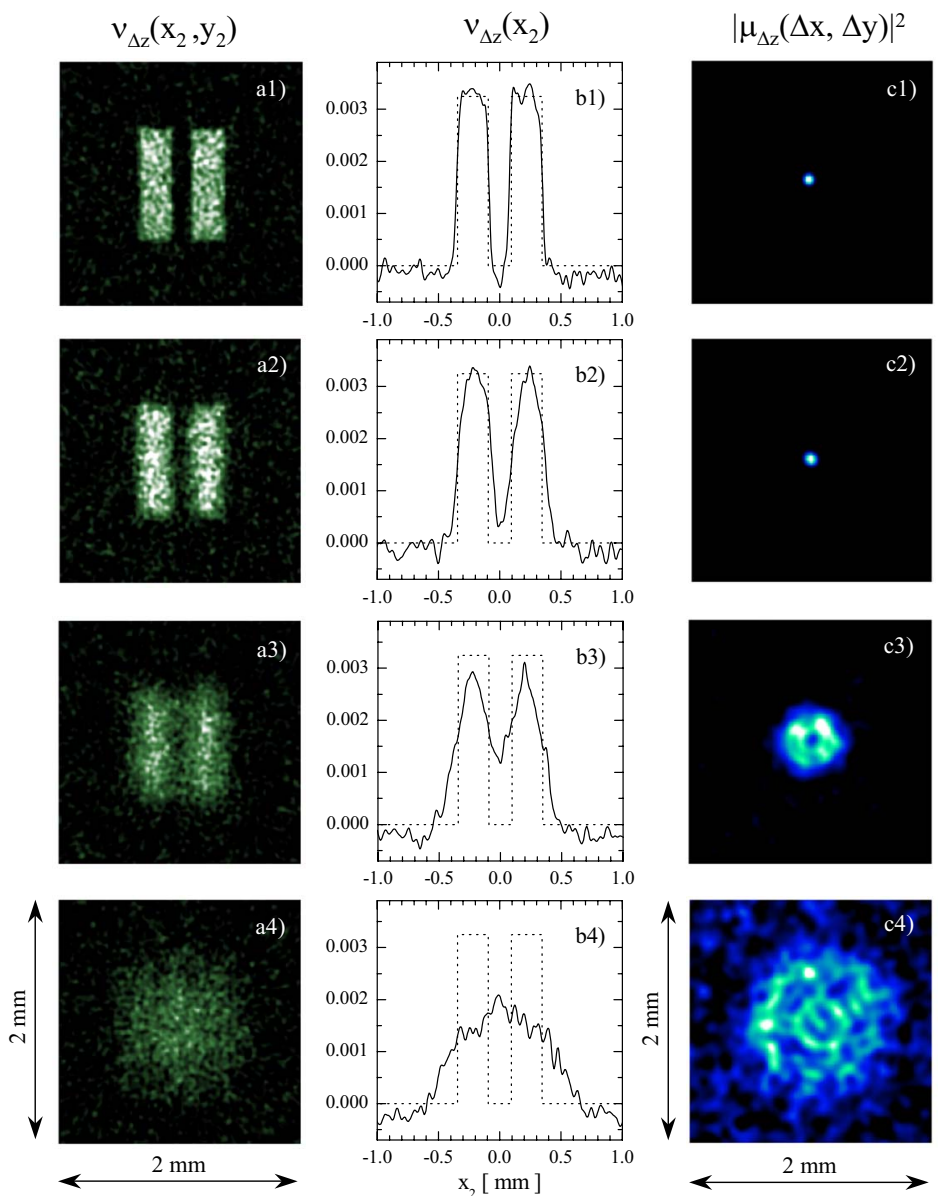
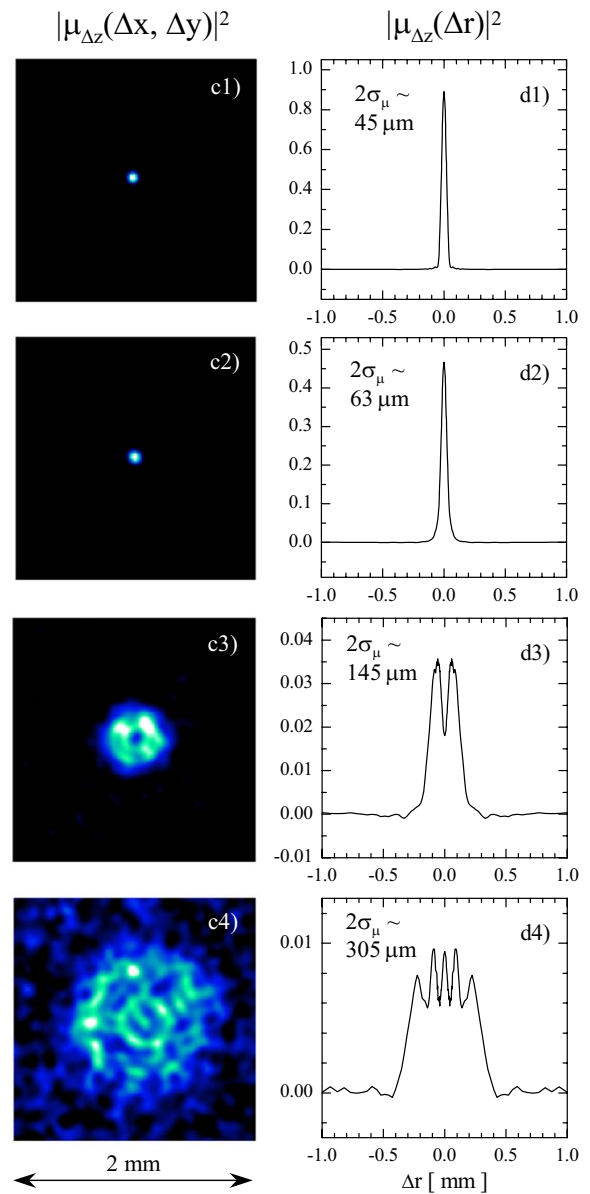

FIG. 2. (Color online) Experimental results obtained at four distances $\Delta z$ $=0$ (raw 1), $\Delta z=10 \mathrm{~mm}$ (raw 2) $\Delta z$ $=30 \mathrm{~mm}$ (raw 3) and $\Delta z=60 \mathrm{~mm}$ (raw 4): panels on column 1 are the 2D-ghost images obtained by averaging $M=10^{4}$ independent realizations of the speckle field; panels on the second column are the horizontal sections of the 2D images averaged over all the lines falling inside the object (continuous lines) and corresponding expected profiles (dotted lines); panels on third column are the 2D-squared correlation coefficients measured between the planes $z_{1}$ and $z_{2}=z_{1}+\Delta z$; panels on the last column show the radial averages of the thirdcolumn panels, reporting also the values of their rms full widths $2 \sigma_{\mu}$. Notice the different vertical scales in panels (d1), (d2), (d3), and (d4).
$\Delta z=10 \mathrm{~mm}$ (second raw of Fig. 2) show that the ghost image is slightly blurred [panels (a2) and (b2)], but characterized by the same visibility as for the case $\Delta z=0$. For $\Delta z=30 \mathrm{~mm}$ (third raw) the image becomes severely blurred [panels (a3) (b3)] and the double slit is barely visible. This is only a classical 2D-convolution effect because, in this case, $2 \sigma_{\mu}$ $\sim 145 \mu \mathrm{m}$ is comparable with the distance $(160 \mu \mathrm{m})$ between the two apertures. Notice also that the visibility is substantially unchanged, in spite of the fact that the amplitude of $\left|\mu_{\Delta z}\right|^{2}$ is only a few percent. For $\Delta z=60 \mathrm{~mm}$ (last raw), the ghost image is completely blurred because, in this case, $2 \sigma_{\mu} \sim 305 \mu \mathrm{m}$. Notice that, although the amplitudes of $\left|\mu_{\Delta z}\right|^{2}$ in panels $(\mathrm{d} 3)$ and $(\mathrm{d} 4)$ are both very small $(\sim 1 \%)$, their radial profiles exhibit neat "Fresnel diffraction" patterns of a round aperture, similar to the point spread function observed out of focus in wide angle optical microscopy. ${ }^{19}$ These aspects, related to the three-dimensional behavior of $\left|\mu_{\Delta z}(\Delta \mathbf{x})\right|^{2}$, will be elucidated in Refs. 15 and 16 .

In conclusion, we have demonstrated that the longitudinal coherence of a speckle beam in lensless thermal ghost imaging is the key ingredient for determining the visibility and the spatial resolution of the retrieved image. We showed that, by operating within the near-field region of a source producing speckles of size $\delta \sim 45 \mu \mathrm{m}$, the disappearance of the ghost image for $z_{1} \neq z_{2}$ is due to the fading out of the intensity-intensity cross correlation between the two planes, and no quantum explanation is necessary. This article provides a key of interpretation for the results, apparently inconsistent one with each other, reported in Refs. 12 and 13.

We thank L. A. Lugiato for discussions and support.

${ }^{1}$ D. V. Strekalov et al., Phys. Rev. Lett. 74, 3600 (1995).

${ }^{2}$ T. B. Pittman et al., Phys. Rev. A 52, R3429 (1995).

${ }^{3}$ A. Gatti et al., Phys. Rev. Lett. 93, 093602 (2004).

${ }^{4}$ R. S. Bennink et al., Phys. Rev. Lett. 89, 113601 (2002).

${ }^{5}$ F. Ferri et al., Phys. Rev. Lett. 94, 183602 (2005).

${ }^{6}$ A. Valencia et al., Phys. Rev. Lett. 94, 063601 (2005).

${ }^{7}$ M. Bache et al., Phys. Rev. A 73, 053802 (2006).

${ }^{8}$ R. S. Bennink et al., Phys. Rev. Lett. 92, 033601 (2004).

${ }^{9}$ A. F. Abouraddy et al., Phys. Rev. Lett. 93, 213903 (2004).

${ }^{10}$ G. Scarcelli, V. Berardi, and Y. Shih, Phys. Rev. Lett. 96, 063602 (2006).

${ }^{11}$ A. Gatti et al., Phys. Rev. Lett. 98, 039301 (2007).

${ }^{12}$ G. Scarcelli, V. Berardi, and Y. Shih, Appl. Phys. Lett. 88, 061106 (2006).

${ }^{13}$ L. Basano and P. Ottonello, Appl. Phys. Lett. 89, 091109 (2006).

${ }^{14}$ J. W. Goodman, Speckle Phenomena in Optics (Roberts, Greenwood Village, CO, 2006), pp. 90-92.

${ }^{15}$ A. Gatti, D. Magatti, and F. Ferri, "3D coherence of light speckles: Theory," (unpublished).

${ }^{16}$ D. Magatti, A. Gatti, and F. Ferri, "3D coherence of light speckles: Experiment," (unpublished).

${ }^{17}$ R. Cerbino, Phys. Rev. A 75, 053815 (2007).

${ }^{18}$ M. Giglio, M. Carpineti, and A. Vailati, Phys. Rev. Lett. 85, 1416 (2000).

${ }^{19}$ M. B. Cannell et al., in Handbook of Biological Confocal Microscopy, edited by J. B. Pawley (Springer, New York, 2006), Chap. 25. 